\section{OPEN ACCESS}

Approved by:

Frontiers Editorial Office,

Frontiers Media SA, Switzerland

*Correspondence:

Aihua Liu

liuaihuadoctor@163.com

Ning Ma

marnine@163.com

†These authors have contributed equally to this work

Specialty section: This article was submitted to

Endovascular and Interventional Neurology,

a section of the journal

Frontiers in Neurology

Received: 02 December 2021 Accepted: 06 December 2021

Published: 05 January 2022

Citation:

Chen J, Tao M, Han J, Feng X Peng F, Tong $X$, Niu $H$, Ma N and Liu A (2022) Corrigendum: Pipeline Embolization Device for the Treatment

of Unruptured Intracranial Dissecting Aneurysms. Front. Neurol. 12:824841.

doi: 10.3389/fneur.2021.824841

\title{
Corrigendum: Pipeline Embolization Device for the Treatment of Unruptured Intracranial Dissecting Aneurysms
}

\begin{abstract}
Jigang Chen ${ }^{1,2+}$, Mushun $\mathrm{Tao}^{3 \dagger}$, Jiangli Han ${ }^{4}$, Xin Feng ${ }^{5}$, Fei Peng ${ }^{1,2}$, Xin Tong ${ }^{1,2}$, Hao Niu ${ }^{1,2}$, Ning $\mathrm{Ma}^{3 *}$ and Aihua Liu ${ }^{1,2 *}$

${ }^{1}$ Beijing Neurosurgical Institute, Capital Medical University, Beijing, China, ${ }^{2}$ Department of Interventional Neuroradiology, Beijing Tiantan Hospital, Capital Medical University, Beijing, China, ${ }^{3}$ Department of Neurosurgery, First Hospital of Shanxi Medical University, Taiyuan, China, ${ }^{4}$ Department of Neurosurgery, The Third Xiangya Hospital, Central South University, Changsha, China, ${ }^{5}$ Department of Neurosurgery Beijing Hospital, National Center of Gerontology, Beijing, China
\end{abstract}

Keywords: unruptured intracranial dissecting aneurysms, pipeline endovascular device, outcomes, complications, treatment

\section{A Corrigendum on}

Pipeline Embolization Device for the Treatment of Unruptured Intracranial Dissecting Aneurysms

by Chen, J., Tao, M., Han, J., Feng, X., Peng, F., Tong, X., Niu, H., Ma, N., and Liu, A. (2021). Front. Neurol. 12:691897. doi: 10.3389/fneur.2021.691897

In the published article, there was an error in affiliation 3, as published. Instead of "Department of Neurosurgery, Third Hospital of Shanxi Medical University, Taiyuan, China," it should be "Department of Neurosurgery, First Hospital of Shanxi Medical University, Taiyuan, China."

The authors apologize for this error and state that this does not change the scientific conclusions of the article in any way. The original article has been updated.

Publisher's Note: All claims expressed in this article are solely those of the authors and do not necessarily represent those of their affiliated organizations, or those of the publisher, the editors and the reviewers. Any product that may be evaluated in this article, or claim that may be made by its manufacturer, is not guaranteed or endorsed by the publisher.

Copyright () 2022 Chen, Tao, Han, Feng, Peng, Tong, Niu, Ma and Liu. This is an open-access article distributed under the terms of the Creative Commons Attribution License (CC BY). The use, distribution or reproduction in other forums is permitted, provided the original author(s) and the copyright owner(s) are credited and that the original publication in this journal is cited, in accordance with accepted academic practice. No use, distribution or reproduction is permitted which does not comply with these terms. 